\title{
Characterization of Aspergillus aculeatus $\beta$-glucosidase 1 accelerating cellulose hydrolysis with Trichoderma cellulase system
}

\author{
Yutaro Baba, Jun-ichi Sumitani, Shuji Tani and Takashi Kawaguchi
}

\begin{abstract}
Aspergillus aculeatus $\beta$-glucosidase 1 (AaBGL1), which promotes cellulose hydrolysis by Trichoderma cellulase system, was characterized and compared some properties to a commercially supplied orthologue in A. niger (AnBGL) to elucidate advantages of recombinant AaBGL1 ( $r A a B G L 1)$ for synergistic effect on Trichoderma enzymes. Steady-state kinetic studies revealed that YAaBGL1 showed high catalytic efficiency towards $\beta$-linked glucooligosaccharides. Up to a degree of polymerization (DP) 3, rAaBGL1 prefered to hydrolyze $\beta-1,3$ linked glucooligosaccharides, but longer than DP 3, preferred $\beta-1,4$ glucooligosaccharides (up to DP 5). This result suggested that there were different formation for subsites in the catalytic cleft of AaBGL1 between $\beta-1,3$ and $\beta-1,4$ glucooligosaccharides, therefore rAaBGL1 preferred short chain of laminarioligosaccharides and long chain of cellooligosaccharides on hydrolysis. rAaBGL1 was more insensitive to glucose inhibition and more efficient to hydrolyze the one of major transglycosylation product, gentiobiose than $\mathrm{AnBGL}$, resulting that $\mathrm{rAaBGL1}$ completely hydrolyzed 5\% cellobiose to glucose faster than AnBGL. These data indicate that AaBGL1 is valuable for the use of cellulosic biomass conversion.
\end{abstract}

Keywords: Aspergillus aculeatus; $\beta$-glucosidase; Biomass conversion; Trichoderma reesei; Cellulase

\section{Introduction}

Cellulosic biomass has been most abundant biomass that widely distributed on earth. Cellulose is degraded into monomeric sugar by cellulases, and is appropriate source of biofuels and biochemicals production. However, high crystallinity and insolubility of cellulose makes difficult to degrade to soluble sugar, such as glucose. Cellulose degradation is accomplished by the synergistic action among endoglucanases (E.C. 3.2.1.4), cellobiohydrolases (E.C. 3.2.1.91), and $\beta$-glucosidases (BGLs, E.C. 3.2.1.21, Woodward 1991). However it has been known that cellobiohydrolases and BGLs are significantly inhibited by the hydrolysis products such as cellobiose and glucose, and that the product inhibition reduces the overall rate of cellulose hydrolysis (Andric et al. 2010; Xiao et al. 2004). BGLs hydrolyze $\beta$-glucosidic bonds to release glucose units from the non-reducing end of $\beta$ glucoologosaccharides or glucosides. BGLs are classified in the glycoside hydrolase (GH) family $1,3,5,9,30$, and

\footnotetext{
* Correspondence: monger@biochem.osakafu-u.ac.jp

Graduate School of Life and Environmental Sciences, Osaka Prefecture

University, 1-1 Gakuen-cho, Naka-ku, Sakai, Osaka 599-8531, Japan
}

116 in the CAZy database (Henrissat 1991; Henrissat and Bairoch 1993,1996; URL: http://www.cazy.org/). In the fungal cellulase system, BGL mainly hydrolyze cellooligosaccharides to glucose on the final step of cellulose degradation. Thus the BGL having high hydrolytic activity is required to forestall the product inhibition by cellobiose against cellobiohydrolases ( $\mathrm{Du}$ et al. 2010). It has been known that the cellulase mixture secreted by the filamentous fungus Trichoderma reesei, which is used for industrial application, has very low activity of BGL, and this problem has been tried to solve by addition of exogenous BGL, for example from A. niger (Berlin et al. 2007; Chauve et al. 2010; Dekker 1986; Singhania et al. 2013). Since the BGL is important for cellulase system, the BGL having more powerful activity for hydrolysis and accelerating cellulase system of $T$. reesei is required to promote saccharification of the cellulosic biomass.

Aspergillus aculeatus no. F-50 [NBRC 108796] was isolated in soil whose cellulose- and hemicellulose-degrading enzymes effectively hydrolyzed pulp in combination with 
T. reesei (Murao et al. 1979). AaBGL1, a dominant BGL in the culture supernatant of $A$. aculeatus no F-50, was purified and characterized (Sakamoto et al. 1985a,b), and its cDNA was cloned and sequenced (Kawaguchi et al. 1996). AaBGL1 has unique features for hydrolysis of cellooligosaccharides in terms of not only showing high specific activity for cellooligosaccharides with increasing degree of polymerization (DP) up to 5 , but also being detected no transglycosylation products on hydrolysis of cellobiose and insoluble cellooligosaccharides by paper chromatography (Sakamoto et al. 1985b; unpublished data). Moreover, Nakazawa et al. reported that $T$. reesei expressing AaBGL1 gene under the control of $x y n 3$ promoter (X3AB1 strain) exhibited 63- and 25-fold higher BGL activity than that of PC-3-7 strain and X3TB1 strain which expressed $T$. reesei BGL I gene under the control of xyn3 promoter respectively (Nakazawa et al. 2011). In addition, JN11, which is the crude cellulase preparation from X3AB1, released more glucose than commercially available cellulases, Accellerase 1500 and Cellic CTec from various pretreated biomasses if those have rich hemicelluloses (e.g. $\mathrm{NaOH}$ pretreatment; Kawai et al. 2012). Kawai et al. mentioned for these results that JN11 has the best balance of BGL and hemicellulase activities for the degradation of cellulosic biomasses. As described above, AaBGL1 is a useful BGL for biomass conversion. However, AaBGL1 has not been investigated the enzymatic analysis in detail.

There are many fungal BGLs which share high similarity of amino acid sequence with AaBGL1 in the GH family 3. Nevertheless, the reason why AaBGL1 was selected for use in cellulosic biomass conversion, was unclear due to partial investigation of the detailed enzymatic properties. Here we demonstrated the availability of rAaBGL1 by characterization of rAaBGL1, especially substrate specificity and transglycosylation products, and comparing capability to hydrolyze cellobiose with BGL from $A$. niger (AnBGL) which shares high amino acid sequence similarity with AaBGL1.

\section{Materials and methods}

\section{Strains and medium}

Escherichia coli DH5 $\alpha \mathrm{F}^{\prime}$ was used as a host for construction of recombinant plasmids. A. oryzae niaD300 strain was used as a host for AaBGL1 gene expression.

A. oryzae transfomant was cultivated at $30^{\circ} \mathrm{C}$ with shaking $(160 \mathrm{rpm})$ in Erlenmeyer flasks in the minimal medium (MM) with 5\% glucose, 1.5\% $\mathrm{NaNO}_{3}, 0.00008 \%$ $\mathrm{Mo}_{7} \mathrm{O}_{24} \cdot 2 \mathrm{H}_{2} \mathrm{O}, 0.00111 \% \mathrm{H}_{3} \mathrm{BO}_{3}, 0.00016 \% \mathrm{CoCl} \cdot 6 \mathrm{H}_{2} \mathrm{O}$, $0.00016 \% \mathrm{CuSO}_{4} \cdot 5 \mathrm{H}_{2} \mathrm{O}, 0.005 \%$ EDTA.2Na, 0.0005\% FeS$\mathrm{O}_{4} \cdot 7 \mathrm{H}_{2} \mathrm{O}, 0.0005 \% \mathrm{MnCl}_{2} \cdot 4 \mathrm{H}_{2} \mathrm{O}, 0.0022 \% \mathrm{ZnSO}_{4} \cdot 7 \mathrm{H}_{2} \mathrm{O}$, $0.00013 \% \mathrm{KCl}, 0.00013 \% \mathrm{MgSO}_{4} \cdot 7 \mathrm{H}_{2} \mathrm{O}, 0.00038 \% \mathrm{KH}_{2} \mathrm{PO}_{4}$ (pH 6.5) for appropriate days.

\section{Expression of AaBGL1 gene in $A$. oryzae and purification of recombinant $A a B G L 1$}

The AaBGL1 gene was amplified by PCR using $A$. aculeatus genomic DNA as a template, with 5'- aactgcaggcggc cgcatcatgaagctcagttggcttg-3' as a sense primer and 5'-aagc atgctcattgcaccttcgggagc- 3 ' as an antisense primer. PCR condition is the following thermal settings: 30 cycles of $10 \mathrm{~s}$ initial denaturation step at $98^{\circ} \mathrm{C}$, followed by $5 \mathrm{~s}$ annealing step at $55^{\circ} \mathrm{C}$, and $3 \mathrm{~min}$ of extension step at $72^{\circ} \mathrm{C}$ using PrimeSTAR HS DNA polymerase (TaKaRa, Japan). The PCR product was digested by Not I and Sph I, and inserted into the same sites of Aspergillus expression vector, pNAN8142 (Minetoki et al. 2003). The resultant plasmid was named as pNPN-AaBGL1. A. oryzae niaD300 strain was transformed with pNAN-AaBGL1 by the protoplast-PEG method (Gomi et al. 1987; Kanamasa et al. 2001). Several transformants was isolated and cultivated to confirm the production of rAaBGL1. The methods of the expression and the purification of rAaBGL1 is previously described (Suzuki et al. 2013). Briefly describing below; A. oryzae transformant overexpressing AaBGL1 gene was cultivated in 2.4 L MM liquid medium for 3 days, and the mycelia were harvested and washed with 5 volume $20 \mathrm{mM}$ sodium acetate buffer ( $\mathrm{pH}$ 5.0). To release rAaBGL1 from cell surface, the mycelia were incubated at $30^{\circ} \mathrm{C}$ for 2 days in $2.4 \mathrm{~L}$ releasing buffer $(10 \mathrm{mg} / \mathrm{ml}$ cycloheximide, $1 \mathrm{mM}$ benzylsulfonyl fluoride, $0.02 \%$ sodium azide in $20 \mathrm{mM}$ sodium acetate buffer ( $\mathrm{pH}$ 5.0)) with shaking. After releasing AaBGL1 from cell surface, supernatant was obtained by filtration as a crude enzyme. The crude enzyme was applied to a DEAE-TOYOPEARL ${ }^{\oplus} 650 \mathrm{M}$ column equilibrated with $20 \mathrm{mM}$ sodium acetate buffer $(\mathrm{pH}$ 5.0). rAaBGL1 was eluted with a linear gradient of $\mathrm{NaCl}(0-0.3 \mathrm{M})$. The active fractions were collected, added to ammonium sulfate at $30 \%$ saturation, and subjected to a Butyl-TOYOPEARL ${ }^{\circ}$ $650 \mathrm{M}$ column equilibrated with $30 \%$ saturation of ammonium sulfate in same buffer. The rAaBGL1 was eluted with a reverse linear gradient of ammonium sulfate (30-0\% saturation). After collecting rAaBGL1 containing fractions, the enzyme was precipitated with ammonium sulfate at $80 \%$ saturation, dissolved in $20 \mathrm{mM}$ sodium acetate buffer ( $\mathrm{pH}$ 5.0), and dialyzed in the same buffer. Homogeneity of rAaBGL1 was confirmed by sodium dodecyl sulfatepolyacrylamide gel electrophoresis (SDS-PAGE).

\section{Purification of BGL from A. niger}

Glucosidase from $A$. niger (SIGMA-ALDRICH, Co.) was dissolved in $5 \mathrm{ml}$ of $20 \mathrm{mM}$ sodium acetate buffer ( $\mathrm{pH}$ 5.0). After dialyzing in the same buffer, the same steps of purification as those of rAaBGL1 were performed (Suzuki et al. 2013), followed by gel filtration on HiLaod $^{\mathrm{TM}} 16 / 60$ Superdex 200 pg column (GE healthcare) equilibrated with $20 \mathrm{mM}$ sodium acetate buffer ( $\mathrm{pH} 5.0)$, and hydrophobic interaction chromatography 
on Hiprep $^{\text {тм }}$ 16/10 Phenyl FF column (low sub; GE healthcare) with reverse linear gradient of ammonium sulfate (30-0\% saturation).

\section{Protein assay}

Protein concentration was determined from the absorbance at $280 \mathrm{~nm}$ using extinction coefficient $(\varepsilon)$ as $162,000 \mathrm{M}^{-1} \cdot \mathrm{cm}^{-1}$ with the exception of the comparison of saccharification between rAaBGL1 and AnBGL.

According to the comparison of BGL ability from $A$. acuelatus and $A$. niger, the protein concentration was determined with the Bio-Rad Protein Assay, based on the method of Bradford (Bio-Rad Laboratories). Bovine $\gamma$-globulin was used as a standard.

\section{Enzyme assays}

Enzymatic reaction was performed by incubating $100 \mu \mathrm{l}$ enzyme with $100 \mu \mathrm{l}$ of $3 \mathrm{mM} p$-nitrophenyl (pNP)-monosaccharides in $100 \mathrm{mM}$ sodium acetate buffer ( $\mathrm{pH} 5.0$ ). Reaction was stopped by adding $2 \mathrm{ml}$ of $1 \mathrm{M} \mathrm{Na}_{2} \mathrm{CO}_{3}$. Released $p$-nitrophenol was quantified by measuring the absorbance at $405 \mathrm{~nm}$ using $\varepsilon$ as $18.5 \mathrm{mM}^{-1} \cdot \mathrm{cm}^{-1}$. One unit of BGL activity was defined as the amount of enzyme required for the release of $1 \mu \mathrm{mol}$ of $p$-nitrophenol per minute from the substrate.

\section{Effect of temperature and $\mathrm{pH}$ on the activity and stability of $r A a B G L 1$}

The optimum temperature was determined by incubating rAaBGL1 (6.13 $\mathrm{nM})$ with $1.5 \mathrm{mM} p$-nitrophenyl- $\beta$ D-glucopyranoside (pNP-Glc) in $20 \mathrm{mM}$ sodium acetate buffer $(\mathrm{pH} 5.0)$ at various temperature $\left(30-70^{\circ} \mathrm{C}\right)$ for $10 \mathrm{~min}$. The optimum $\mathrm{pH}$ was determined by incubating rAaBGL1 (6.13 nM) with $1.5 \mathrm{mM}$ pNP-Glc in various $\mathrm{pH}$ range of buffer (3.3-6.3, sodium citrate buffer; 6.57.3, sodium phosphate buffer; 7.0-8.9, Tris- $\mathrm{HCl}$ buffer; $8.8-10.7$, glycine- $\mathrm{NaOH}$ buffer) at $37^{\circ} \mathrm{C}$ for $10 \mathrm{~min}$. The thermal stability was determined by incubating rAaBGL1 $(6.13 \mathrm{nM})$ at various temperature $\left(30-70^{\circ} \mathrm{C}\right)$ for $30 \mathrm{~min}$. After incubation, sample was on ice for $5 \mathrm{~min}$, followed by incubating with $1.5 \mathrm{mM}$ pNP-Glc in $20 \mathrm{mM}$ sodium acetate buffer $\left(\mathrm{pH} \mathrm{5.0)}\right.$ at $37^{\circ} \mathrm{C}$ for $10 \mathrm{~min}$. The $\mathrm{pH}$ stability was determined by incubating rAaBGL1 (61.3 nM) in $100 \mathrm{mM}$ various $\mathrm{pH}$ range of buffer $(1.8-3.2$, glycine- $\mathrm{HCl}$ buffer; 3.4-6.0, sodium acetate buffer; 6.5-7.3, sodium phosphate buffer; 7.2-9.1, Tris- $\mathrm{HCl}$ buffer; 8.8-10.5, glycine- $\mathrm{NaOH}$ buffer) at room temperature for $1 \mathrm{~h}$. After incubation, sample was diluted 10 fold by adding $200 \mathrm{mM}$ sodium acetate buffer ( $\mathrm{pH}$ 5.0), followed by incubation with $1.5 \mathrm{mM}$ pNP-Glc in $20 \mathrm{mM}$ sodium acetate buffer (pH 5.0) at $37^{\circ} \mathrm{C}$ for $10 \mathrm{~min}$.

\section{Inhibition by glucose}

For determination of inhibition constant for glucose on AaBGL1, enzymatic reaction was performed by incubation of rAaBGL1 with $0.1,0.2,0.3$ and $0.4 \mathrm{mM}$ pNP-Glc in the presence of 1.0, 2.5, 5.0, 10.0, 20.0 and $40.0 \mathrm{mM}$ glucose in $20 \mathrm{mM}$ sodium acetate buffer ( $\mathrm{pH} 5.0$ ) at $37^{\circ} \mathrm{C}$. Initial rate of released $p$-nitrophenol were measured, and then, $K_{\mathrm{i}}$ value for glucose on AaBGL1 was calculated with Dixon plot.

For the effect of inhibition by glucose for rAaBGL1 and AnBGL, enzymatic reaction was performed by incubating each enzyme with $1.5 \mathrm{mM}$ pNP-Glc in the presence of $0.05,0.25,0.5,1.0,2.0$, and $4.0 \%$ glucose in $100 \mathrm{mM}$ sodium acetate buffer $(\mathrm{pH} 5.0)$ at $37^{\circ} \mathrm{C}$ for $10 \mathrm{~min}$.

\section{Detection of reaction products by HPAEC-PAD}

For detection of enzymatic reaction products, highperformance anion exchange column chromatography (HPAEC) with a pulsed amperometoric detector (PAD) equipped with a CarboPac PA10 guard column $(4 \times$ $50 \mathrm{~mm})$ and a CarboPac PA10 analytical column $(4 \times$ $250 \mathrm{~mm}$; Dionex Co.) was used. Enzymatic reaction was performed by incubation with equivalent volume of rAaBGL1 (20.0 nM) and each substrate in $20 \mathrm{mM}$ sodium acetate buffer ( $\mathrm{pH} 5.0)$ at $37^{\circ} \mathrm{C}$. Reaction mixture was sampled at appropriate time, and added into equal volume of $0.2 \mathrm{M} \mathrm{NaOH}$. Resultant mixtures were subjected to HPAEC-PAD using mobile phase of $100 \mathrm{mM} \mathrm{NaOH}$ with $10 \mathrm{mM}$ sodium acetate. Glucose, cellobiose (Wako Pure Chemical Industries, Ltd.), cellotriose, cellotetraose, cellopentaose, laminaribiose, laminaritriose, laminaritetraose, laminaripentase (Megazyme), gentiobiose, sophorose (SIGMAALDRICH, Co.) were used as standards.

\section{Kinetic analysis}

For the kinetic analysis, cellobiose, cellotriose, cellotetraose, cellopentaose, lamianaribiose, laminaritriose, laminaritetraose, laminaripentaose and gentiobiose were used as substrates. Appropriate concentrations of each substrate were mixed with equivalent volume of enzyme in $20 \mathrm{mM}$ sodium acetate buffer ( $\mathrm{pH} 5.0$ ). Every 1 or $2 \mathrm{~min}$, reaction was stopped by adding $50 \mu \mathrm{l}$ of $1 \mathrm{~N} \mathrm{HCl}$, and after $5 \mathrm{~min}$, neutralized with adding $50 \mu \mathrm{l}$ of neutralizton solution $(0.4 \mathrm{~N} \mathrm{NaOH}$ and $0.8 \mathrm{M}$ Tris). The amount of released glucose was determined by using Glucose CII-Test Wako (Wako Pure Chemical Industries, Ltd.). Kinetic constants were determined using Hanes-Woolf plot according to Michaelis-Menten equation. In the case of disaccharides, $k_{\text {cat }}$ value was calculated by half of glucose production velocity because one glucodisaccharide molecule composed 2 glucose molecules. Equivalent molar of glucose $\left(G_{1}\right)$ and $G_{n-1}$ production from $G_{n}(n=$ 3-5) was confirmed at the end point of the reaction by HPAEC-PAD. 
For the detection of reaction products by rAaBGL1, laminaribiose, cellobiose, and gentiobiose $(25 \mathrm{mM})$ were reacted with rAaBGL1 $(10.0 \mathrm{nM})$ in $10 \mathrm{mM}$ sodium acetate buffer $(\mathrm{pH} 5.0)$ at $37^{\circ} \mathrm{C}$. Reaction was stopped by addition of equivalent volume of $0.2 \mathrm{~N} \mathrm{NaOH}$ and reaction mixtures were analyzed for HPEAC-PAD as described above.

\section{Results}

\section{Purification and characterization of rAaBGL1}

To investigate the biochemical characterization of rAaBGL1, we purified rAaBGL1 as described in the Materials and methods, and confirmed the homogeneity by SDS-PAGE (Figure 1). The molecular mass calculated from the amino acid sequence was $91.3 \mathrm{kDa}$, however purified rAaBGL1 was approximately $130 \mathrm{kDa}$.

Enzymatic properties of rAaBGL1 were determined using pNP-Glc as a substrate. The enzyme was stable between $40-50^{\circ} \mathrm{C}$, and in a $\mathrm{pH}$ range of $3.0-10.0$ with over $80 \%$ of its maxmum activity. The optimum temperature was $65^{\circ} \mathrm{C}$, and optimum $\mathrm{pH}$ was 5.5 (Figure 2).

Specific activity for several pNP-monosaccharides was determined (Table 1). rAaBGL1 was shown highest activity toward pNP-Glc, and slight activity toward pNP- $\beta-\mathrm{D}-$ fucopyranoside, $\mathrm{pNP}-\alpha-\mathrm{L}$-arabinofuranoside, $\mathrm{pNP}-\beta-\mathrm{D}-\mathrm{xy}-$ lopyranoside, $\mathrm{pNP}-\beta-\mathrm{D}$-galactopyranoside. No activity was detected for $\mathrm{pNP}-\beta-\mathrm{D}$-mannopyranoside, $\mathrm{pNP}-\mathrm{N}$-acetyl- $\beta$ D-glucosaminide.

Substrate specificity and kinetic parameters for natural $\beta$-glucooligosaccharides was determined (Table 2). For disaccharide hydrolysis, rAaBGL1 was shown the highest $k_{\text {cat }} / K_{\mathrm{m}}$ value toward laminaribiose among three disaccharides because of the lowest $K_{\mathrm{m}}$ value. Cellobiose was not preferable substrate for rAaBGL1 because of the highest $K_{\mathrm{m}}$ value and lowest $k_{\text {cat }}$ value. The $k_{\text {cat } /} K_{\mathrm{m}}$ value for cellooligosaccharides and laminarioligosaccharides were increased up to tetra- and trisaccharide, respectively. AaBGL1 exhibited stationary high $k_{\text {cat }}$ value for cellopentaose, whereas displayed the lower affinity and turnover number for laminaritetraose and laminaripentaose than laminaritriose.

\section{Detection of transglycosylation product}

To identify the transglycosylation products by rAaBGL1, the time course of the reaction products using cellobiose, gentiobiose, and laminaribiose as a substrate were analyzed by HPAEC-PAD (Figure 3 ). In the early stage of the reaction $(0-1 \mathrm{~h})$, the reaction product of rAaBGL1 with each substrate was glucose. In the middle stage of the reaction $(2-4 \mathrm{~h})$, the reaction products of rAaBGL1 with cellobiose and laminaribiose were glucose and gentiobiose from transglycosylation (Figure 3A,C). In the reaction with gentiobiose, it is expected that gentiobiose was produced as a transglycosylation product as in the

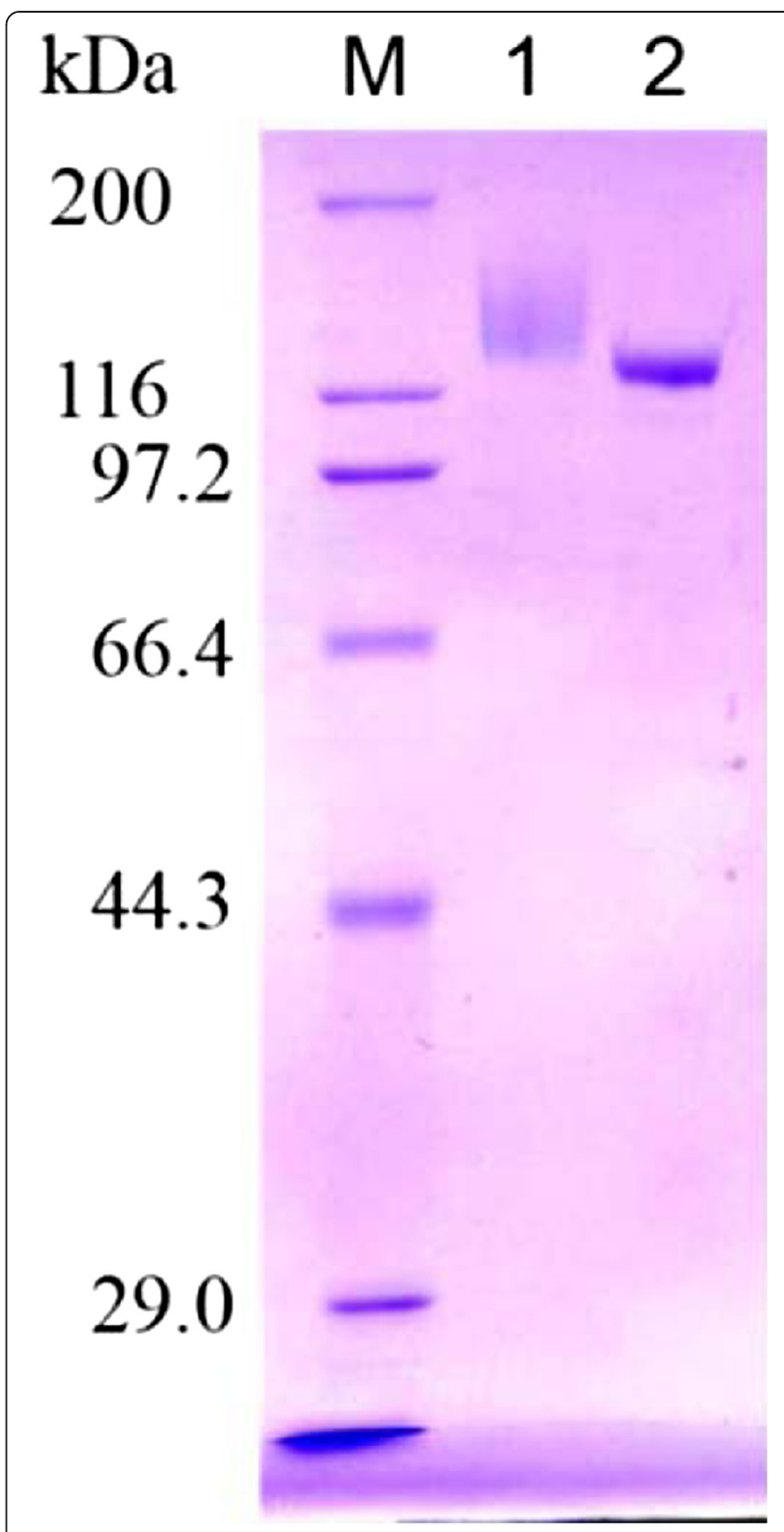

Figure 1 Purification of $\mathrm{rAaBGL1}$ and AnBGL. M, molecular weight markaer; 1, AaBGL1; 2, AnBGL.

case with cellobiose and laminaribiose, because any oligosaccharides other than gentiobiose were not detected (Figure 3B). Thus, these results indicated that gentiobiose was only or main product of transglycosylation by rAaBGL1 under the condition used in this study. In the final stage, the reaction product of $\mathrm{rAaBGL1}$ with each substrate was glucose because of the high hydrolytic activity toward a transglycosylation product, gentiobiose (Table 2).

\section{Comparison of the saccharification ability between} rAaBGL1 and AnBGL on the hydrolysis of $5 \%$ cellobiose To evaluate the performance of rAaBGL1, AnBGL which shared 82.4\% identity with AaBGL1 (Dan et al. 2000; 
A
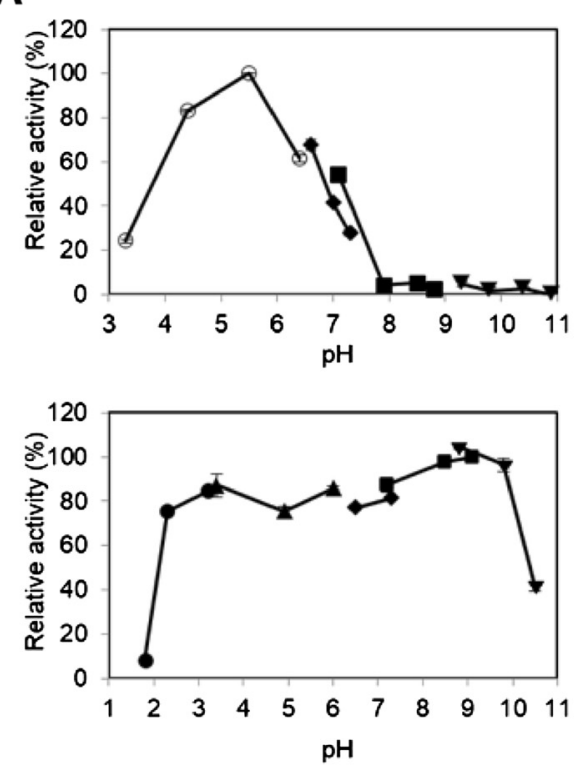

B
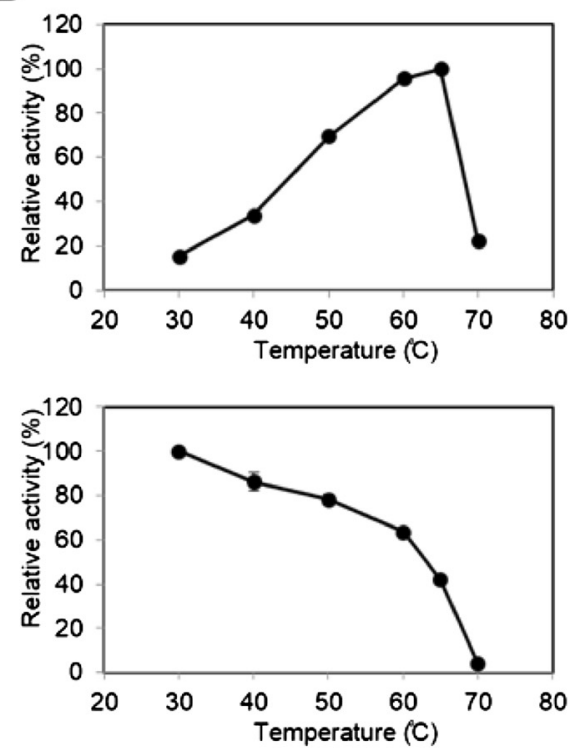

Figure 2 Effects of $\mathrm{pH}(\mathrm{A})$ and temperature (B) on the activity (upper panels) and the stability (lower panels) of purified rAaBGL1. To determine the effect of $\mathrm{pH}$ on the activity and the stability (A), enzyme was incubated with $1.5 \mathrm{mM}$ pNP-Glc for 10 min in $100 \mathrm{mM}$ following buffers: glycine-HCl, pH 1.9-2.8 (closed circle); sodium acetate, pH 3.4-5.9 (closed triangle); sodium citrate, pH 3.3-6.3 (open circle); sodium phosphate, pH 6.4-7.3 (closed diamond); Tris-HCl pH 6.8-8.9 (closed square); Glycine- $\mathrm{NaOH}$ pH 9.5-11.0 (closed inveted triangle). To determine the effect of temperature on the activity and the stability (B), enzyme was incubated with $1.5 \mathrm{mM}$ pNP-Glc at 30-70ْ in $100 \mathrm{mM}$ sodium acetate buffer ( $\mathrm{pH}$ 5.0). Data are expressed at the mean \pm the standard deviation of three independent experiments.

Seidle et al. 2004) was selected. AnBGL from Sigmaaldrich was purified to homogeneity (Figure 1), and compared the hydrolysis of $5 \%$ cellobiose with rAaBGL1 (Figure 4). rAaBGL1 produced glucose faster than AnBGL through the entire reaction time measured. rAaBGL hydrolyzed almost completely $5 \%$ cellobiose after $8 \mathrm{~h}$ reaction $(94.1 \pm 0.8 \%)$, but AnBGL was not sufficient $(82.8 \pm 0.4 \%)$.

\section{Glucose inhibition}

Generally, BGL is inhibited by the reaction product, glucose. Therefore we compared the sensitivity to various concentration of glucose on the hydrolysis of pNP-Glc between rAaBGL1 and AnBGL (Figure 5). As a result,

Table 1 Specific activity of $\mathrm{rAaBGL} 1$ for various $p$-nitrophenyl- $\beta$-D-glycopyranosides

\begin{tabular}{ll}
\hline Substrate & Specific activity $(\mathbf{U} / \mathbf{m g})$ \\
\hline$p$-nitrophenyl-a-L-arabinofuranoside & 0.057 \\
$p$-nitrophenyl- $\beta$-D-fucopyranoside & 0.017 \\
$p$-nitrophenyl- $\beta$-D-xylopyranoside & 0.428 \\
$p$-nitrophenyl- $\beta$-D-glucopyranoside & 128 \\
$p$-nitrophenyl- $\beta$-D-galactopyranoside & 0.006 \\
$p$-nitrophenyl- $\beta$-D-mannopyranoside & $\mathrm{ND}$ \\
$p$-nitrophenyl- $N$-acetyl- $\beta-D$-glucosaminide & $\mathrm{ND}$ \\
\hline
\end{tabular}

ND:Not Detected.
rAaBGL1 was lower sensitivity to glucose at all the concentration tested than AnBGL. The $K_{\mathrm{i}}$ value for gluose on rAaBGL1 used pNP-Glc as a substrate was $9.99 \pm$ $0.94 \mathrm{mM}$ (data not shown).

\section{Discussion}

In this study, we performed detailed investigation of enzymatic properties of rAaBGL1 that have already demonstrated synergistic effects by adding to the cellulase system of $T$. reesei.

Table 2 Kinetic parameters of rAaBGL1 for various natural substrates

\begin{tabular}{lllc}
\hline Substrate & $\boldsymbol{K}_{\mathbf{m}}(\mathbf{m M})$ & $\boldsymbol{k}_{\text {cat }}\left(\mathbf{s}^{\mathbf{- 1}}\right)$ & $\boldsymbol{k}_{\mathbf{c a t}} / \boldsymbol{K}_{\mathbf{m}}\left(\mathbf{s}^{\left.\mathbf{- 1} \cdot \mathbf{m M}^{-\mathbf{1}}\right)}\right.$ \\
\hline gentiobiose & $0.52 \pm 0.02$ & $457 \pm 4$ & $873 \pm 29$ \\
laminaribiose & $0.41 \pm 0.02$ & $444 \pm 17$ & $1080 \pm 20$ \\
laminaritriose & $0.22 \pm 0.01$ & $337 \pm 3$ & $1550 \pm 80$ \\
laminaritetraose & $0.72 \pm 0.03$ & $304 \pm 4$ & $423 \pm 11$ \\
laminaripentaose & $1.13 \pm 0.01$ & $285 \pm 5$ & $251 \pm 2$ \\
cellobiose & $2.06 \pm 0.07$ & $354 \pm 10$ & $172 \pm 3$ \\
cellotriose & $0.45 \pm 0.02$ & $477 \pm 3$ & $1060 \pm 50$ \\
cellotetraose & $0.32 \pm 0.01$ & $433 \pm 4$ & $1340 \pm 40$ \\
cellopentaose & $0.41 \pm 0.02$ & $433 \pm 9$ & $1070 \pm 30$ \\
\hline
\end{tabular}

Each value is the mean of triplicate experiments. 

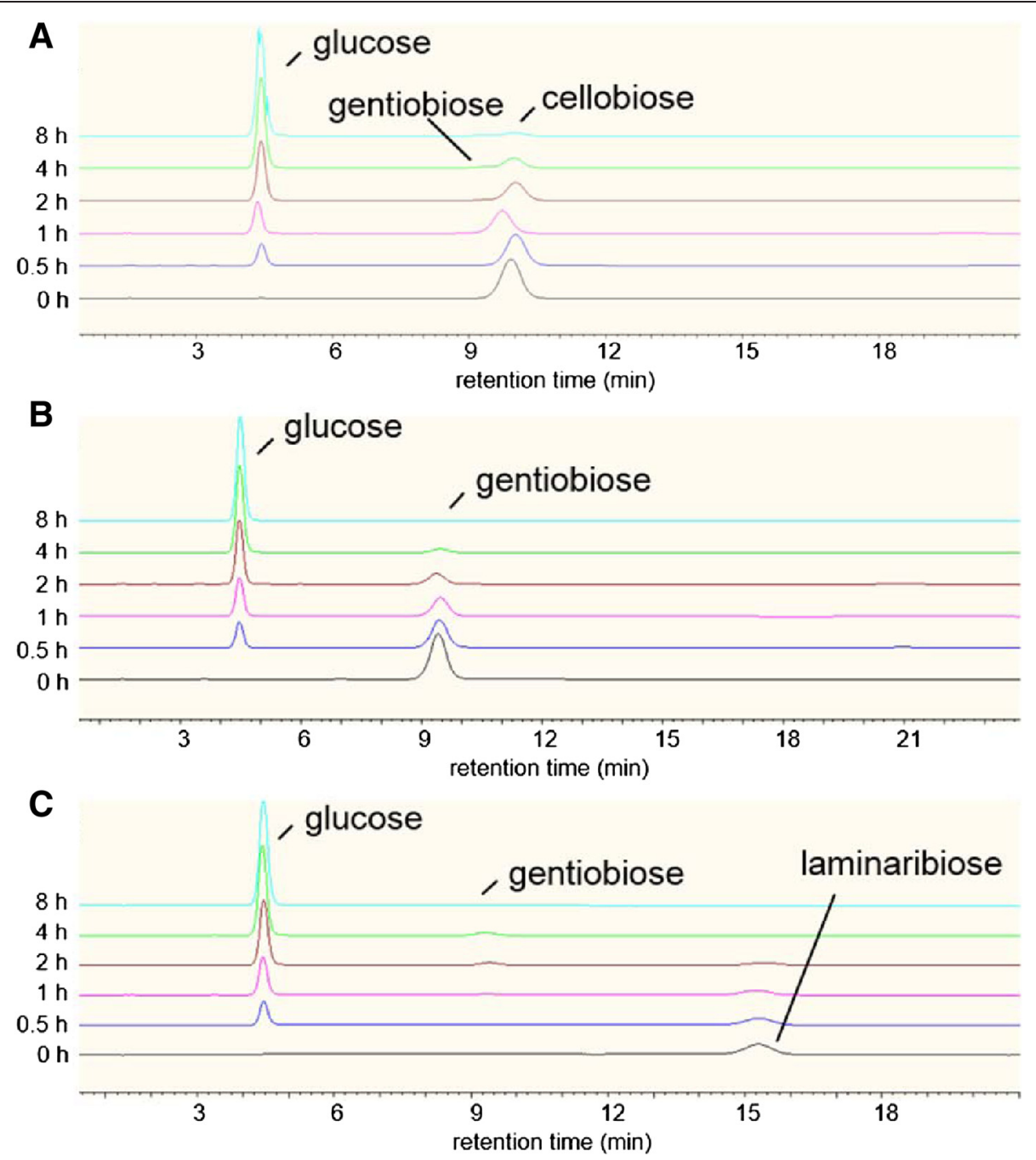

Figure 3 Hydrolysis of disaccharides by rAaBGL1. The hydrolysis of $25 \mathrm{mM}$ cellobiose (A), gentiobiose (B), and laminaribiose (C) was performed by incubation with $10.0 \mathrm{nM} \mathrm{AaBGL} 1$ at $37^{\circ} \mathrm{C}$ for $8 \mathrm{~h}$. The hydrolysis products of indicating times were analyzed by HPAEC-PAD, and identified by comparison of retention time of each peak with those of standards.

Mature AaBGL1 was consisted 841 amino acids, and confirmed secretion in culture supernatant due to possessing signal peptide. The molecular mass calculated from the amino acid sequence was $91.3 \mathrm{kDa}$, however, purified rAaBGL1 showed approximately $130 \mathrm{kDa}$ by SDS-PAGE analysis (Figure 1). This molecular mass was similar to native AaBGL1 in culture supernatant from A. aculeatus (Sakamoto et al. 1985a). Recently, crystalline structure of rAaBGL1, which treated with the endoglycosidase $\mathrm{H}$ at undenaturing condition was solved at a $1.80 \AA$ resolution (Suzuki et al. 2013). AaBGL1 has $9 N$-glycans out of 16 potential $\mathrm{N}$-glycosylation sites in the monomer, and $\mathrm{O}$-glycosylation was not observed. BGLs from A. kawachii, $A$. niger and $A$. oryzae that shared high similarity of amino acid sequence with AaBGL1 have more than 10 potential $N$-glycosylation sites in their amino acid sequence and occur several $\mathrm{N}$-glycosylations, in consequence, these BGLs indicated the similar molecular mass with AaBGL1 (Iwashita et al. 1998, 1999; Langston et al. 2006; Seidle et al. 2004).

In previous study, enzymatic properties of authentic AaBGL1 were investigated (Sakamoto et al. 1985b). Thus, we compared enzymatic properties of AaBGL1 between recombinant protein from $A$. oryzae and authentic one. Thermal and $\mathrm{pH}$ profiles were almost similar between authentic and recombinant AaBGL1 with exception that rAaBGL1 had higher optimum temperature and thermal stability, and wider range of $\mathrm{pH}$ stability than authentic AaBGL1, although assay conditions were different. BGL2, which is probably isoform of AaBGL1 generated by different glycosylation, had higher stability than BGL1. The slight differences of thermal and $\mathrm{pH}$ 


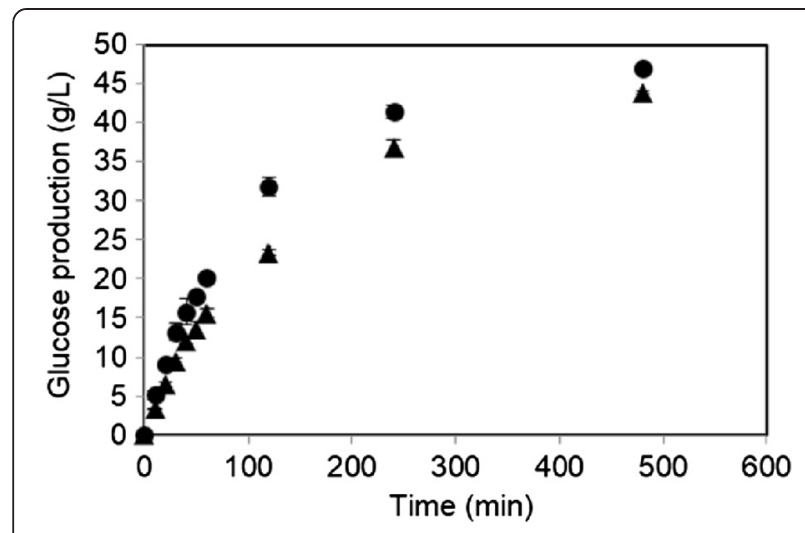

Figure 4 Comparison of the ability of $5 \%$ cellobiose hydrolysis between rAaBGL1 (closed circle) and AnBGL (closed triangle). Each enzyme was incubated with $5 \%$ cellobiose. Released glucose was measured by glucose oxidase method. Data are expressed at the mean \pm the standard deviation of three independent experiments.

profiles between authentic and recombinant AaBGL1 might be developed from the difference of modification by glycosylation between $A$. aculeatus and A. oryzae.

It is known that GH family 3 is composed of $\beta$ glucosidase (EC 3.2.1.21), glucan 1,3- $\beta$-glucosidase (EC 3.2.1.58), glucan $1,4-\beta$-glucosidase (EC 3.2.1.74), exo1,3-1,4-glucanase (EC 3.2.1.-), xylan 1,4- $\beta$-xylosidase (EC 3.2.1.37), $\beta-N$-acetylhexosaminidase (EC 3.2.1.52), $\alpha$-L-arabinofuranosidase (EC 3.2.1.55). In addition, BGLs are divided into three groups on the basis of their substrate specificity, such as aryl- $\beta$-glucosidase, aryland alkyl- $\beta$-glucosidase, and enzymes with broad substrate specificity (Takahashi et al. 2011). Moreover, Harvey et al. classified GH family 3 BGLs into 6 distinct

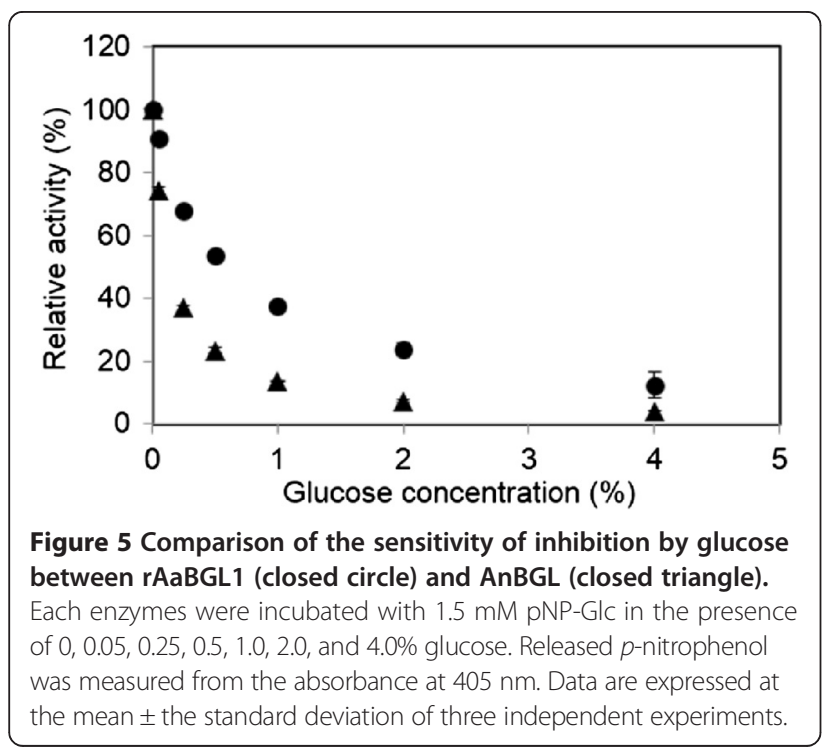

phylogenetic branches by their amino acid sequence similarities (Harvey et al. 2000), and Suzuki et al. mentioned that diverse structures of the subsite +1 makes different substrate specificity in GH family 3 BGLs (Suzuki et al. 2013). However, substrate specificity of AaBGL1 has been investigated only for $\beta-1,4$ linked glucan and oligosaccharides (Sakamoto et al. 1985b). Therefore, in this study, the detailed substrate specificity of AaBGL1 was investigated. As a result, rAaBGL1 exhibited high specificity toward pNP-Glc, that is $\beta$-glucosidic linkage (Table 1$)$. In kinetic analysis for natural disaccharides, catalytic efficiency increased in the order of cellobiose $<$ gentiobiose $<$ laminaribiose among three $\beta$-disaccharides. In all substrates used in this study, rAaBGL1 exhibited highest catalytic efficiency $\left(k_{\text {cat }} / K_{\mathrm{m}}\right)$ for laminaritriose due to lowest $K_{\mathrm{m}}$ value, but longer than laminaritriose, $k_{\text {cat }} / K_{\mathrm{m}}$ value was decreased because of lowering the affinity and the turnover number. Eukaryotic GH family 3 BGLs have been characterized that there is a tendency for laminaribiose to be the best substrate among the other $\beta$-linked disaccharides (Hrmova et al. 2002; Igarashi et al. 2003; Langston et al. 2006; Nakajima et al. 2012; Seidle et al. 2004; Takahashi et al. 2011). On the other hand, for cellooligosaccharides, cellotetraose was the best substrate for rAaBGL1 owing to the stationary low $K_{\mathrm{m}}$ value and high $k_{\text {cat }}$ value. In addition, several kinetic analyses of GH 3 BGLs imply that there are three subsites, $-1,+1,+2$, in the active site, and that subsite +1 is the most important subsite for the substrate binding (Hrmova et al. 2002; Kawai et al. 2004; Nakatani et al. 2010; Yazaki et al. 1997). Subsite map determined by kinetic analysis of rAaBGL1 for cellooligosaccharides hydrolysis, using the method for subsite analysis of exo-acting enzyme (Hiromi et al. 1973), revealed that AaBGL1 had the subsite +2 like other GH family 3 BGLs, but subsite map for laminarioligosaccharides, showed weak affinity at subsite +2 (Figure 6). Previously, it was demonstrated that

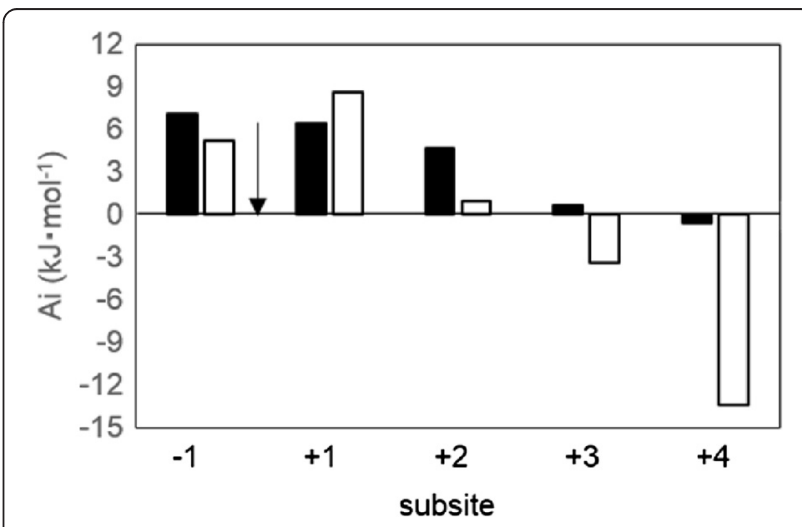

Figure 6 Subsite affinity map of $\mathrm{rAaBGL1}$ for cellooligosaccharides (black bar) and laminarioligosaccharides (white bar), calculated using the $\boldsymbol{K}_{\mathrm{m}}$ and the $\boldsymbol{k}_{\mathrm{cat}}$ values. Arrow indicated the cleavage site. 
AaBGL1 had long, aromatic residue-rich cleft in the active site (Suzuki et al. 2013), and these results suggested that this structure contributed to the activity for long chain (DP 4-6) of cellooligosaccharides, but not of laminarioligosaccharides. Thus, rAaBGL1 preferred short chain (DP 2-3) of laminarioligosaccharides and long chain (DP 4-6) of cellooligosaccharides on hydrolysis.

It has not been clear whether rAaBGL1 has transglycosylation activity because of its potent saccharifying activity. During hydrolysis of gentiobiose, we could not detect any oligosaccharide except for substrate (Figure 3B). On the other hand, gentiobiose was detected in the middle stage of hydrolysis for cellobiose and laminaribiose as a substrate (Figure 3A,C). These results indicated that rAaBGL1 has transglycosylation activity, and can produce gentiobiose at least. This result is consistent with the report the glucose molecule at subsite +1 points its C6 hydroxyl to $\mathrm{C} 1$ hydroxyl of the glucose molecule at subsite -1 in AaBGL1-glucose complex (Suzuki et al. 2013). In fact, it has been reported that BGL from A. niger also produces gentiobiose as one of transglycosylation products (Seidle et al. 2004). Moreover, it has been reported that some BGLs transferred glucose from non-reducing end of substrate to $\mathrm{O} 6$ position of non-reducing end of $\beta$-linked disaccharides (Kawai et al. 2004; Kono et al. 1999; Seidle et al. 2004; Watanabe et al. 1992). However, we could not clearly identify to produce trisaccharide as a transglycosylation product by rAaBGL1 under the condition used in this study.

In cellulosic biomass hydrolysis using Trichoderma cellulase system, cellobiose is accumulated by the dominant enzyme, cellobiohydrolase I that plays an important role for the hydrolysis of crystalline cellulose and by low activity of BGLs. The accumulation of cellobiose causes the lowering of overall activity of cellulase system via the inhibition of cellobiohydrolase activity. Therefore, we attempted to compare the saccharifying activity toward cellobiose between rAaBGL1 and commercially available AnBGL, an orthologue of AaBGL1 (82.4\% identity). The optimum $\mathrm{pH}$ of AaBGL1 and AnBGL was around 4. (Sakamoto et al. 1985b, Seidle et al. 2004). In this study, we compared the cellobiose hydrolytic activity at $\mathrm{pH}$ 5.0, because enzymatic saccharification of cellulosic biomasses was performed at $\mathrm{pH}$ between $4.8-5.0$ in the many studies (Berlin et al. 2007; Chen et al. 2013; Dashtban and Qin 2012; Harrison et al. 2013; Kawai et al. 2012). As a result, rAaBGL1 hydrolyzed 5\% cellobiose faster than AnBGL (Figure 4), because of the difference of the sensitivity for the product inhibition, especially by glucose. In fact, rAaBGL1 was more insensitive to inhibition by glucose than AnBGL at all the glucose concentration tested (Figure 5 ). $K_{\mathrm{i}}$ value for glucose of AaBGL1 is $9.99 \pm 0.94 \mathrm{mM}$, on the other hand, that of AnBGL was $3 \mathrm{mM}$ (Seidle et al. 2004). Moreover, the specific activity for a major transglycosylation product, gentiobiose of rAaBGL1 and AnBGL was $170 \pm 4 \mathrm{U} / \mathrm{mg}$ and $57 \pm 1 \mathrm{U} / \mathrm{mg}$, respectively, under the condition of $25 \mathrm{mM}$ gentiobiose in $10 \mathrm{mM}$ sodium acetate buffer (pH 5.0) at $37^{\circ} \mathrm{C}$ (data not shown). AnBGL exhibited higher $K_{\mathrm{m}}$ value $(1.3 \mathrm{mM})$ toward gentiobiose than AaBGL1 $(0.52 \mathrm{mM})$, although $K_{\mathrm{m}}$ value toward cellooligosaccharides of AnBGL was almost similar to AaBGL1 at each optimum pH condition (Seidle et al. 2004). Thus, we concluded that AaBGL1 has high saccharifying activity toward cellobiose compared with AnBGL, because AaBGL1 was insensitive to competitive inhibition by glucose, and has high hydrolytic activity toward gentiobiose, which is transglycosylation product during cellobiose hydrolysis. Recently, the saccahrifying activity of JN11, a crude enzyme preparation from $T$. reesei $\mathrm{X} 3 \mathrm{AB} 1$ strain expressing AaBGL1 gene was compared with those of commercially available cellulase, Accellerase 1500 and Cellic CTec using various types of pretreated biomass as substrates (Kawai et al. 2012). Using JN11, especially glucose yield was improved, suggesting that the advantage of JN11 for saccharification of cellulosic biomass resulted in the increase of cellobiase activity by AaBGL1.

In this study, we investigated the biochemical characterization of rAaBGL1 from Aspergillus acuelatus no. F-50 strain, and compared the cellobiose-saccharifying activity between rAaBGL1 and AnBGL, an orthologue of AaBGL1. Here we demonstrated the potent cellobiase activity of rAaBGL1 for cellulosic biomass degradation, is combined with the high hydrolyzing efficiency toward gentiobiose derived from transglycosylation and low sensitivity to the product inhibition by glucose.

\section{Competing interests}

The authors declare that they have no competing interests.

\section{Authors' contributions}

YB: Performed the experiments for purification and characterization of the enzymes, analyzed data, and drafted manuscript. JS: Conceived the study, designed the experiments, critically analyzed data, and revised manuscript. ST: Performed experiments for DNA manipulation and gene expression. TK: Designed the experiments, fritically analyzed data, and revised manuscript. All authors read and approved the final manuscript.

\section{Acknowledgements}

This work was financially supported by The New Energy and Industrial Technology Deveropment Organization and by Grant-in-Aid for Scientific Reserch (22580090) from the Japan Society for the Promotion of Science.

Received: 25 November 2014 Accepted: 23 December 2014 Published online: 24 January 2015

\section{References}

Andric P, Meyer AS, Jensen PA, Johansen KD (2010) Reactor design for minimizing product inhibition during enzymatic lignocellulose hydrolysis: I. Significance and mechanism of cellobiose and glucose inhibition on cellulolytic enzymes. Biotechnol Adv 28:308-324

Berlin A, Maximenko V, Gilkes N, Saddler J (2007) Optimization of enzyme complexes for lignocellulose hydrolysis. Biotechnol Bioeng 97:287-296

Chauve M, Mathis H, Huc D, Casanave D, Monot F, Ferreira NL (2010) Comparative kinetic analysis of two fungal $\beta$-glucosidases. Biotechnol Biofuels 3:3 
Chen Y, Stevens MA, Zhu Y, Holmes J, Xu H (2013) Understanding of alkaline pretreatment parameters for corn stover enzymatic saccharification. Biotechnol Biofuels 6:8

Dan S, Marton I, Dekel M, Bravdo BA, He S, Withers SG, Shoseyov O (2000) Cloning, expression, characterization, and nucleophile identification of family 3, Aspergillus niger $\beta$-glucosidase. J Biol Chem 275:4973-4980

Dashtban M, Qin W (2012) Overexpression of an exotic thermotolerant $\beta$-glucosidase in trichoderma reesei and its significant increase in cellulolytic activity and saccharification of barley straw. Microb Cell Fact 11:63

Dekker RF (1986) Kinetic, inhibition, and stability properties of a commercial $\beta$-D-glucosidase (cellobiase) preparation from Aspergillus niger and its suitability in the hydrolysis of lignocellulose. Biotechnol Bioeng 28:1438-1442

Du F, Wolger E, Wallace L, Liu A, Kaper T, Kelemen B (2010) Determination of product inhibition of $\mathrm{CBH} 1, \mathrm{CBH} 2$, and $\mathrm{EG} 1$ using a novel cellulase activity assay. Appl Biochem Biotechnol 161:313-317

Gomi K, limura Y, Hara S (1987) Integrative transformation of Aspergillus oryzae with a plasmid containing the Aspergillus nidulans argB gene. Agric Biol Chem 51:2549-2555

Harrison MD, Zhang Z, Shand K, O'Hara IM, Doherty WOS, Dale JL (2013) Effect of pretreatment on saccharification of sugar cane bagasse by complex and simple enzyme mixtures. Bioresour Technol 148:105-113

Harvey AJ, Hrmova M, De Gori R, Varghese JN, Fincher GB (2000) Comparative modeling of the three-dimensional structures of family 3 glycoside hydrolases. Proteins 41:257-269

Henrissat B (1991) A classification of glycosyl hydrolases based on amino-acid sequence similarities. Biochem J 280:309-316

Henrissat B, Bairoch A (1993) New families in the classification of glycosyl hydrolases based on amino- acid sequence similarities. Biochem J 293:781-788

Henrissat B, Bairoch A (1996) Updating the sequence-based classification of glycosyl hydrolases. Biochem J 316:695-696

Hiromi K, Niita Y, Numata C, Ono S (1973) Subsite affinities of glucoamylase: examination of the validity of the substie theory. Biochim Biophys Acta 302:362-375

Hrmova M, Gori RD, Smith BJ, Fairweather JK, Driguez H, Varghese JN, Fincher GB (2002) Structural basis for broad substrate specificity in higher plant $\beta$-D-glucan glucohydrolases. Plant Cell 14:1033-1052

Igarashi K, Tani T, Kawai R, Samejima M (2003) Family 3 -glucosidase from cellulose-degrading culture of the white-rot fungus Phanerochaete chrysosporium is a glucan 1,3- $\beta$-glucosidase. J Biosci Bioeng 95:572-576

Iwashita K, Todoroki K, Kimura H, Shimoi H, Ito K (1998) Purification and characterization of extracellular and cell wall bound $\beta$-glucosidases from Aspergillus kawachii. Biosci Biotechnol Biochem 62:1938-1946

Iwashita K, Todoroki K, Kimura H, Shimoi H, Ito K (1999) The bglA gene of Aspergillus kawachii encodes both extracellular and cell wall-bound ß-glucosidases. Appl Environ Micorbiol 65:5546-5553

Kanamasa S, Takada G, Kawaguchi T, Sumitani J, Arai M (2001) Overexpression and purification of Aspergillus aculeatus $\beta$-mannosidase and analysis of the integrated gene in Aspergillus oryzae. J Biosci Bioeng 92:131-137

Kawaguchi T, Enoki T, Tsurumaki S, Sumitani J, Ueda M, Ooi T, Arai M (1996) Cloning and sequencing of the cDNA encoding beta-glucosidase 1 from Aspergillus aculeatus. Gene 173:287-288

Kawai R, Igarashi K, Kitaoka M, Ishii T, Samejima M (2004) Kinetics of substrate transglycosylation by glycoside hydrolase family 3 glucan $(1 \rightarrow 3)$ - $\beta$-glucosidase from the white-rot fungus Phanerochaete chrysosporium. Carbohydr Res 339:2851-2857

Kawai T, Nakazawa H, Ida N, Okada H, Tani S, Sumitani J, Kawaguchi T, Ogasawara W, Morikawa Y, Kobayashi Y (2012) Analysis of the saccharification capability of high-functional cellulase JN11 for various pretreated biomasses through a comparison with commercially available counterparts. J Ind Microbiol Biotechnol 39:1741-1749

Kono H, Kawano S, Tajima K, Erata T, Takai M (1999) Structural analyses of new tri- and tetrasaccharides produced from disaccharides by transglycosylation of purified Trichoderma viride $\beta$-glucosidase. Glycoconj J 16:415-423

Langston J, Sheehy N, Xu F (2006) Substrate specificity of Aspergillus oryzae family 3 B-glucosidase. Biochim Biophys Acta 1764:972-978

Minetoki T, Tsuboi H, Koda A, Ozeki K (2003) Development of high expression system with the improved promoter using the cis-acting element in Aspergillus species. J Biol Macromol 3:89-96

Murao S, Kanamoto J, Arai M (1979) Isolation and identification of a cellulolytic enzyme producing microorganism. J Ferment Technol 57:151-156
Nakajima M, Yamashita T, Takahashi M, Nakano Y, Takeda T (2012) Identification, cloning, and characterization of $\beta$-glucosidase from Ustilago esculenta. Appl Microbiol Biotechnol 93:1989-1998

Nakatani Y, Lamont IL, Cutfield JF (2010) Discovery and characterization of a distinctive Exo-1,3/1,4- $\beta$-glucanase from the marine bacterium Pseudoalteromonas sp. strain BB1. Appl Enviorn Micorbiol 76:6760-6768

Nakazawa H, Kawai T, Ida N, Shida Y, Kobayashi Y, Okada H, Tani S, Sumitani J, Kawaguchi T, Morikawa Y, Ogasawara W (2011) Construction of a recombinant Trichoderma reesei strain expressing Aspergillus aculeatus $\beta$-glucosidase 1 for efficient biomass conversion. Biotechnol Bioeng 109:92-99

Sakamoto R, Arai M, Murao S (1985a) Purification and Physicochemical Properties of Three $\beta$-Glucosidases from Aspergillus aculeatus No. F-50. Agric Biol Chem 49:1275-1281

Sakamoto R, Arai M, Murao S (1985b) Enzymic properties of three $\beta$-glucosidases from Aspergillus aculeatus No F-50. Agric Biol Chem 49:1283-1290

Seidle HF, Marten I, Shoseyov O, Huber RE (2004) Physical and kinetic properties of the family $3 \beta$-glucosidase from Aspergillus niger which is important for cellulose breakdown. Protein J 23:11-23

Singhania RR, Patel AK, Sukumaran RK, LarrocheC PA (2013) Role and significance of $\beta$-glucosidases in the hydrolysis of cellulose for bioethanol production. Bioresour Technol 127:500-507

Suzuki K, Sumitani J, Nam Y, Toru N, Tani S, Wakagi T, Kawaguchi T, Fushinobu S (2013) Crystal structures of glycoside hydrolase family $3 \beta$-glucosidase 1 from Aspergillus aculeatus. Biochem J 452:211-221

Takahashi M, Konishi T, Takeda T (2011) Biochemical characterization of Magnaporthe oryzae $\beta$-glucosidases for efficient $\beta$-glucan hydrolysis. Appl Microbiol Biotechnol 91:1073-1082

Watanabe T, Sato T, Yoshioka S, Koshijima T, Kuwahara M (1992) Purification and properties of Aspergillus niger ß-glucosidase. Eur J Biochem 209:651-659

Woodward J (1991) Synergism in cellulase systems. Bioresource Technol 36:67-75

Xiao Z, Zhang X, Gregg DJ, Saddler JN (2004) Effects of sugar inhibition on cellulases and $\beta$-glucosidase during enzymatic hydrolysis of softwood substrates. Appl Biochem Biotechnol 113-116:1115-1126

Yazaki T, Ohnishi M, Rokushika S, Okada G (1997) Subsite structure of the $\beta$-glucosidase from Aspergillus niger, evaluated by steady-state kinetics with cello-oligosaccahrides as substrates. Carbohydr Res 298:51-57

\section{Submit your manuscript to a SpringerOpen ${ }^{\odot}$ journal and benefit from:}

- Convenient online submission

- Rigorous peer review

- Immediate publication on acceptance

- Open access: articles freely available online

- High visibility within the field

- Retaining the copyright to your article

Submit your next manuscript at springeropen.com 